\title{
Effect of Intravenous Fentanyl to Prevent Radial Artery Spasm During Trans Radial Intervention
}

\author{
PARTHA PRATIM SAHA ${ }^{1}$, AMAL KUMAR CHOUDHURY ${ }^{1}$, SYED ALI AHSAN ${ }^{2}$, ABDUL MOMEN ${ }^{1}$, SHAFIQUR \\ RAHMAN PATWARY ${ }^{1}$, NUR ALAM ${ }^{1}$, KOFIL UDDIN ${ }^{1}$, PRITHA SARKAR ${ }^{3}$, HERU AL AMIN ${ }^{1}$ \\ ${ }^{1}$ Department of Cardiology, National Institute of Cardiovascular Diseases (NICVD), Dhaka, Bangladesh, ${ }^{2}$ Department of Cardiology, Bangabandhu \\ Sheikh Mujib Medical University, Shahbag, Dhaka, Bangladesh (BSMMU), Dhaka, Bangladesh, ${ }^{3} \mathrm{KBFGH}$, Dhaka, Bangladesh. \\ Corrospondence : Dr.Partha Pratim Saha. Resident, NICVD, E-mail: parthadmc.62@gmail.com
}

\begin{abstract}
Background: Radial artery spasm (RAS), one of the commonest complications in trans radial cardiac intervention, leads a variety range of adverse events. Different prevention strategies had been introduced and the use of sedation is one of them. Use of Fentanyl is common in cardiac interventions to achieve sedation and analgesia and also offers a potent vasodilatory effect.

Objective: This study aimed to explore the role of fentanyl in the prevention of $R A S$ in trans radial intervention.

Methods: This observational analytic study was conducted in National Institute of Cardiovascular Diseases (NICVD) for a period of 1 year following approval of the protocol in the total of 260 patients who underwent coronary intervention through trans radial approach (Coronary Angiogram and or Percutaneous Coronary Intervention). They were categorized in 2 group- group I patients received intravenous fentanyl and group II patients didn't receive fentanyl. Besides this, each patient received all treatments according to standard protocol of NICVD. RAS was identified by subjective and objective evidence. Patient is discomfort was quantified with a visual analogue scale.

Results: The frequency of RAS was $17.70 \%$ in group I versus $30.80 \%$ in group II $(p=0.014)$. Intravenous use of fentanyl significantly reduced radial artery spasm $(p<0.05)$. Bivariate logistic regression analysis showed use of fentanyl had lower odds to prevent radial artery spasm (odds ratio [OR]: 0.430, 95\% confidence interval [CI]: 0.239 to 0.779). The number needed to treat to avoid 1 case of spasm was 8 . Multivariate logistic regression analysis also revealed use of fentanyl was the strong predictor of RAS ([OR]: 0.370; 95\% [CI]: .195-.703) as well as use of reprocessed sheath, smoking, catheter diameter 6Fr and visual analogue scale (VAS) Score during cannulation became independent predictaor of RAS. Patient discomfort in VAS score was $4.75 \pm 2.70$ in Group I versus $5.33 \pm 2.64$ in group II $(p<0.05)$.

Conclusion: In this study, use of fentanyl reduced the frequency of radial artery spasm and the procedure related level of patient discomfort.
\end{abstract}

Key words: Radial artery spasm (RAS), Fentanyl, Coronary Intervention (CAG and or PCI)

University Heart Journal 2022; 18(1): 54-60

Introduction:

Cardiovascular disease (CVD) remains the most common cause of death worldwide. CAG is the gold standard method for the diagnosis and for establishing treatment strategies. ${ }^{1}$

CAG can be performed via femoral, radial, brachial, ulnar or axillary arteries. Due to drawbacks of TFA, coronary procedure through trans-radial approach (TRA) has been adopted. Through the TRA the first CAG was performed by Lucian Campeau in $1989^{2}$ and first PCI was performed in 1992 by Ferdinand Kiemeneij. ${ }^{3}$ The radial artery has become the preferred choice due to easy compressibility and distance from major veins and nerves, accompanying blood flow through the ulnar artery to the palmar arch, improved time to ambulation and shorter hospitalization duration, ${ }^{4}$ additional comfort to patient, lower hospital expenses, ${ }^{5}$ and improve clinical outcomes including survival by reducing potentially life-threatening complications. That's why TRA for coronary procedure is gaining momentum as an alternative. Although TRA in coronary intervention is well established, it has disadvantages too like somewhat challenging, difficult to master, and requires more learning curve. ${ }^{6}$ Trans-radial specific vascular complications are radial artery spasm (RAS), compartment syndrome, radial artery occlusion (RAO). Other complications of TRA include bleeding, hematoma, perforation, laceration, dissection, avulsion of artery, arteriovenous fistula, pseudoaneurysm, digital ischemia. $^{7}$

RAS can be defined as a temporary, sudden, narrowing of the radial artery. ${ }^{8}$ It is usually diagnosed clinically and angiographically during cardiac intervention. The reported incidence of RAS during TRA ranged from $4 \%$ to $20 \% \cdot 9,10$ 
The big range in the rate of incidence could be accounted partly by different types of intraarterial vasodilatory cocktails used in different cardiac intervention laboratories. Predictors of spasm includes female gender, smaller wrist circumference, lower body mass index, tobacco abuse, vaso occlusive disorder, ad hoc coronary interventions, $>2$ catheters during a procedure, $>6$ French catheters, smaller intra-luminal radial artery diameter, operator inexperience, traumatic puncture, radial artery to sheath ratio $<1$, increased procedure duration and radial artery anatomic anomalies ${ }^{11}$ and catheter properties, including coating and length. ${ }^{12}$

Fentanyl, a potent synthetic $\mu$ receptor- stimulating opioid, has become one of the most used drugs for both procedural analgesia and sedation in patients undergoing CAG and or PCI. Routine administration of relatively low doses of an opioid and benzodiazepine combination during trans-radial interventional procedures is associated with a substantial reduction in the rate of spasm, the need for access site crossover, and the procedure-related level of patient discomfort. The absence of peri-and post-procedural complications represents the starting point of future research about preventive use of fentanyl not only for sedation purposes but also to prevent arterial spasm. The present study was conducted to find out the efficacy of fentanyl to prevent RAS during trans radial intervention.

\section{Study Methods:}

This prospective observational analytic study was conducted in NICVD from January, 2020 to December, 2020 on patients who underwent coronay procedure through TRA with positive modified Allen's test. Objective of the study was evaluation of the efficacy of intravenous fentanyl during trans radial coronary intervention to prevent the radial artery spasm. Patient with history of radial and/or ulnar artery injury and surgery, acute heart failure, congestive cardiac failure, cardiogenic shock, end stage renal disease having arterio-venous fistula or planned for making arterio-venous fistula, known peripheral arterial disease of upper limb, non palpable radial artery/ ipsilateral ulnar artery, prior CABG surgery using radial artery, abnormal liver function test and chronic opioid and benzodiazepine use were excluded from the study. RAS was the dependent and use of fentanyl was the independent variable.

\section{Radial artery spasm (RAS) $)^{13}$ : Clinical RAS}

The operator assessed RAS on the basis of a questionnaire addressing the following five sign

- Persistent forearm pain,

- Pain response to catheter manipulation,

- Pain response to sheath insertion and withdrawal,

- Difficult catheter manipulation after being "trapped" by radial artery,

- Considerable resistance on withdrawal of the sheath.

Patients who had at least 2 of the 5 signs or by the presence of just 1 when it is necessary to administer a second dose of spasmolytic agent were diagnosed as clinical RAS.

\section{Angiographic RAS}

- Angiographic RAS was divided into mild $(<30 \%)$, moderate $(30 \%-70 \%)$ and severe $(>70 \%)$ spasm by radial artery angiography. Moderate and severe spasm were defined as angiographic RAS.

- The diagnostic criterion was the combination of clinical and or angiographic RAS. RAS was diagnosed by at least two experienced operators.

A total of 260 patients were enrolled in this study according to inclusion and exclusion criteria among 260 patients. 130 patients were included in group I and 130 patients were included in group II. After reviewing the most current vital signs, only the group I patients were given 25 microgram IV fentanyl slowly over 2 minutes at the beginning of the procedure (during preparation of the access site). One additional dose was allowed $60 \mathrm{~min}$ after the first one. Selection of patient of this group was done by opertaor's own discretion. Arm rest was provided with the table, so that the patients arm was in abduction. A 500 $\mathrm{ml}$ plastic sachet of normal saline was placed on the armrest beneath the wrist to elevate the wrist. The hand was then fixed in hyperextension with adhesive tape, prepared in a sterile fashion and the draped positioned. Pre-procedure ipsilateral radial pulse strength $(0-4+)$ was documented for all (4+ Bounding, 3+ Increased, 2+ Normal, 1+ Weak, 0+ Absent or nonpalpable). Vitals signs were checked every 30 minutes ( \pm 10 minutes) after application of the intravenous fentanyl for 60 minutes $( \pm 10$ minutes) then every 60 minutes ( \pm 10 minutes) up to the end of the procedure in all patients. The respiration and oxygen saturation of the patient were constantly monitored by a dedicated interventional trained nurse. Angiographic views of radial artery obtained just after sheath insertion 
and just before sheath withdrawal or any time during difficulties in wire and catheter manipulation for patients of both groups. After sterile preparation, 0.5 - $1.0 \mathrm{ml}$ of subcutaneous lidocaine was administered and radial artery was cannulated using modified seldinger technique with help of vasofix and intraarterial line was established by 6 Fr vascular access sheath. Parenteral radial cocktail (Intra arterial 200 microgram GTN + intra arterial $2.5 \mathrm{mg}$ verapamil) was given with exact combination and dose at direction of operator based on patient's hemodynamic status. Repeat use of parenteral radial cocktail was made when there was difficulty in moving catheters, when patients complained of persistent forearm pain, and when operators found resistance during catheter and vascular access sheath introduction as well as withdrawal. Along with that 5,000 unit of unfractionated heparin for CAG and 10,000 unit of unfractionated heparin for PCI were administered during the procedure in the contralateral hand. During PCI activated clotting time (ACT) values were kept between 250-300 sec with additional heparin if needed. Over the 0.032 inch exchange wire, the diagnostic catheter (most commonly Tiger catheter) was advanced to the ascending aorta using fluoroscopic guidance to do CAG. Judgkin Right (JR)/Judgkin Left (JL)/ Extra Back Up (EBU) guide catheter were used in cases of PCI. The choice of guidewires and catheters was made by the operator. Parameters were documented. When difficulty was encountered in advancing the wire from the subclavian artery to the ascending aorta, the patient was asked to take a deep breath. The radial artery sheath was removed immediately after completion of procedure, and haemostasis was achieved by conventional hemostatic method with rolled gauge and leucoband. Conventional haemostatic compression was removed when adequate hemostasis was achieved usually after two hours. Patient's perceived peri-procedure forearm discomfort documented in the morning after the procedure with a visual analogue scale (VAS) whereby patient graded the discomfort on a scale of 0 to 10 (0 corresponding to "no discomfort" and 10 to "extreme pain and discomfort"). Post-procedure strength of ipsilateral radial pulse (0-4+) after radial band removal and presence and size of hematoma at access site at time of radial band removal were documented. All data were collected through a structured questionnaire.

\section{Statistical Methods:}

The Statistical Package for Social Sciences (SPSS) version 21 software was used for data analysis. Categorical variables were expressed as absolute number and percentages and continuous variables as mean and standard deviation. Categorical variables were compared through chi-square test. Continuous variables were compared through unpaired student t test. Univariate logistic regression analysis as well as multivariate regression analysis was done to identify the predictors of RAS. A pvalue $<0.05$ was considered statistically significant.

\section{Results:}

Majority of the respondents were in age group 41-50 years old (33.8\% in group I and $38.5 \%$ in group II) with a mean age of $56.21 \pm 7.17$ and $55.55 \pm 7.32$ (SD). No statistical difference was observed between two groups $(\mathrm{p}=0.457)$. Majority of the patients were male $(63.10 \%$ in group I and $73.10 \%$ in group II). No statistical difference was observed between both group $(\mathrm{p}=0.08)$. In group I, $51.50 \%$ underwent Percutaneous Coronary Intervention (PCI) and 49.50\% underwent Coronary Angiography (CAG). In group II, 50.80\% patients underwent PCI and 49.20\% patients underwent $\mathrm{CAG}$.

No statistical difference was observed between group I and group II considering procedure performed $(\mathrm{p}=0.901)$. Presenting risk factors among study population were in descending order hypertension $(91.9 \%)$, dyslipidemia $(49.3 \%)$, diabetes mellitus $(37.7 \%)$, active smoking $(21.9 \%)$, renal dysfunction $(15 \%)$, peripheral artery disease (14.6\%) and family history of CAD (12.30\%). Two group did not have any significant statistical difference ( $p$ $>0.05)$ considering coronary artery disease risk factors. No statistical difference was observed between two groups $(\mathrm{p}>0.05)$ in relation with BMI, height and weight. Mean BMI in Group I was 25.86 \pm 2.87 and Group II was $25.82 \pm 2.66$. In $91.9 \%$ of patients, right radial artery access was used and left radial artery access was used in $8.1 \%$ of patients with no statistical difference. Mean VAS score during cannulation was $4.75 \pm 2.70$ and $5.33 \pm 2.64$ among group I and group II accordingly. Pain score was significantly less $(\mathrm{p}=.046)$ in group I patients. Self-reported patient discomfort was significantly lower in group I. Spasm was, expectedly, strongly associated with higher patient discomfort. About $10.76 \%$ of patients had anatomical anomaly, and $9.61 \%$ had subclavian tortuosity with similar distribution in both groups. No. of catheter was $\leq 2$ in $71.2 \%$ of patients and catheter diameter was 5 France in $51.53 \%$ of patients and no significant difference was observed between two groups. New sheath was used in $45.76 \%$ and re processed sheath was used in $54.23 \%$ of patients with similar distribution in both groups. Post dilatation mean arterial pressure was similar in both groups. 
Table-I

Features of procedure underwent among study population:

\begin{tabular}{lcccc}
\hline & Group I & Group II & Total & p value \\
\hline Access & & & & \\
Right radial artery* & $122(83.8)$ & $118(90)$ & $239(91.9)$ & $.255 !$ \\
Left radial artery* & $8(6.2)$ & $13(10)$ & $21(8.1)$ & \\
VAS score of pain & $4.75 \pm 2.70$ & $5.33 \pm 2.64$ & $5.10 \pm 2.56$ & $.046 ! !$ \\
during cannulation** & & & & \\
Anatomical anomaly* & $15(11.53)$ & $12(9.23)$ & $28(10.76)$ & $.472 !$ \\
Subclavian tortuosity* & $16(12.30)$ & $9(6.92)$ & $25(9.61)$ & $.323 !$ \\
No. of catheter* & & & & \\
$\leq 2$ & $99(76.2)$ & $86(66.2)$ & $185(71.2)$ & $.075 !$ \\
$>2$ & $31(23.8)$ & $44(33.8)$ & $75(28.8)$ & \\
Catheter diameter* & & & & \\
5 & $68(52.30)$ & $66(50.76)$ & $134(51.53)$ & .451 \\
6 & $62(47.70)$ & $64(49.24)$ & $126(48.47)$ & \\
Baseline MAP** (mmHg) & $95.39 \pm 3.83$ & $95.59 \pm 3.78$ & $95.48 \pm 3.80$ & $.421 ! !$ \\
Used sheath* & & & \\
New sheath & $58(44.61)$ & $61(46.92)$ & $119(45.76)$ & \\
Re-processed sheath & $72(55.38)$ & $69(53.07)$ & $141(54.23)$ & $.643 !$ \\
Postdialation MAP** (mmHg) & $86 \pm 3.42$ & $85.56 \pm 3.07$ & 85.82 & $.231 ! !$ \\
Length of procedure* (Min) & & & & \\
$\leq 30$ minute & $39(30)$ & $25(19.2)$ & $64(24.6)$ & $.044 !$ \\
$>30$ minute & $91(70)$ & $105(80.8)$ & $196(75.4)$ & \\
Mean \pm SD & $46.42 \pm 13.13$ & $50.31 \pm 12.90$ & $52.30 \pm 8.45$ & $.017 ! !$ \\
Arterial prick before arterial access & & & & \\
Single & $60(46.2)$ & $56(43.1)$ & $116(44.6)$ & \\
Multiple & $70(53.8)$ & $74(56.9)$ & $144(55.4)$ & $.618 !$ \\
\hline
\end{tabular}

The procedure was statistically significantly lengthy in group II patients $(\mathrm{p}=.017)$. Single arterial prick in group I and group II were $46.2 \% \%$ and $43.1 \%$ respectively. There was no significant statistical difference observed regarding single or multiple arterial prick in between two group $(\mathrm{p}=$ 0.618). In group I \& II, $23 \& 40$ patients had RAS respectively. The frequency of radial artery spasm was significantly higher $(30.80 \%)$ in group II versus group I $(17.70 \%)(p=0.014)$. Absolute Risk of RAS in Group I and II was 0.177 and 0.308 respectively. Aboslute Risk Reduction or Risk Difference of RAS $=0.308-0.177=$ 0.131 . So, addition of fentanyl will reduce $13 \%$ more RAS than standard protocol. NNT (Number of patients needed to treat): $1 / 0.131=7.63=8$. So, number needed to treat to avoid 1 case of RAS was 8 . Staistically significant difference was observed regarding severity of RAS in between 2 group $(p=.016)$. Group II patients had more severe radial artery spasm. Group II patients needed more frequent usage of spasmolytic cocktail than group I. In group I, $12.30 \%$ required repeat use of spasmolytic cocktail. In group II, $22.30 \%$ required repeat spasmolytic cocktail. Group II required statistically more repeat use of spasmolytic cocktail $(p=.003)$. Odds ratio of risk factors for occurrence of radial artery spasm (RAS) in the study population by bivariate logistic regression showed not using of fentanyl [OR .430,95\% CI (.239-.779), $\mathrm{p}<.05]$, PCI as procedure [ OR 2.614, 95\% CI (1.448-5.714), $\mathrm{p}<.05$ ], female gender [OR 2.654, 95\% CI (1.470-4.791), $\mathrm{p}<.05$ ], diabetes mellitus [OR 16.724, 95\% CI (7.866$35.560), \mathrm{p}<.05]$, dyslipidemia [OR 4.680,95\% CI (2.4548.927)], use of reprocessed sheath [OR $2.780,95 \%$ CI (1.262-5.038), $\mathrm{p}<.05]$, VAS score during cannulation [OR $11.077,95 \%$ CI (4.031-21.1950), $\mathrm{p}<.05]$, catheter number $>2$ [OR 9.133, 95\% CI (3.990-9.829), $\mathrm{p}<.05$ ], catheter diameter (6Fr) [OR 2.073, 95\% CI (1.016-3.855), $\mathrm{p}<$ 0.05], multiple arterial prick [OR 2.516, 95\% CI (1.403$2.532), \mathrm{p}<.05]$, decreasing height of patient [ OR .857, $95 \%$ CI (.773-.953), $p<0.05]$ and increasing duration of procedure [OR 6.657, 95\% CI (1.584-9.740), $\mathrm{p}<.05$ ] had significantly higher odds for occurring radial artery spasm. 
Table-II

Predictor of RAS in study population

\begin{tabular}{lccc}
\hline Risk Factors & OR & $\begin{array}{c}95 \% \text { CI } \\
\text { Lower limit-Upper limit }\end{array}$ & p value* \\
\hline Use of IV Fentanyl & & $.195-.703$ & .002 \\
Type of procedure (PCI) & .370 & $.498-1.412$ & .508 \\
Female gender & .838 & $.706-2.054$ & .495 \\
Diabetes Mellitus & 1.204 & $.548-1.932$ & .929 \\
Smoking & 1.029 & $1.073-4.633$ & .032 \\
Dyslipidemia & 2.230 & $.706-2.054$ & .495 \\
Catheter number $>2$ & 1.204 & $.515-3.476$ & .549 \\
Catheter diameter 6Fr & 1.338 & $1.118-1.99$ & .048 \\
Increasing VAS score during cannulation & 1.938 & $1.825-2.183$ & .018 \\
Duration of procedure & 1.988 & $.981-1.035$ & .566 \\
Use of reprocessed sheath & 1.008 & $1.349-4.121$ & .024 \\
Height & 1.181 & $.552-1.231$ & .072 \\
Multiple arterial prick & .691 & $.756-25.922$ & .099 \\
\hline
\end{tabular}

Not using of fentanyl [OR .370, 95\% CI (.195-.703), $\mathrm{p}<0.05]$, catheter diameter $6 \mathrm{Fr}$ [OR $1.938,95 \% \mathrm{CI}(1.118$ 1.99), $\mathrm{p}<.05$ ], use of reprocessed sheath [ OR $1.181,95 \%$ CI (1.349-4.121), $\mathrm{p}<.05]$, smoking [OR 2.230, 95\% CI (1.073-4.633), $\mathrm{p}=.032]$ and VAS score during cannulation [OR 1.988, 95\% CI $(1.825-2.183), \mathrm{p}<0.05]$ were independent predictors of radial artery spasm by multivariate logistic regression analysis.

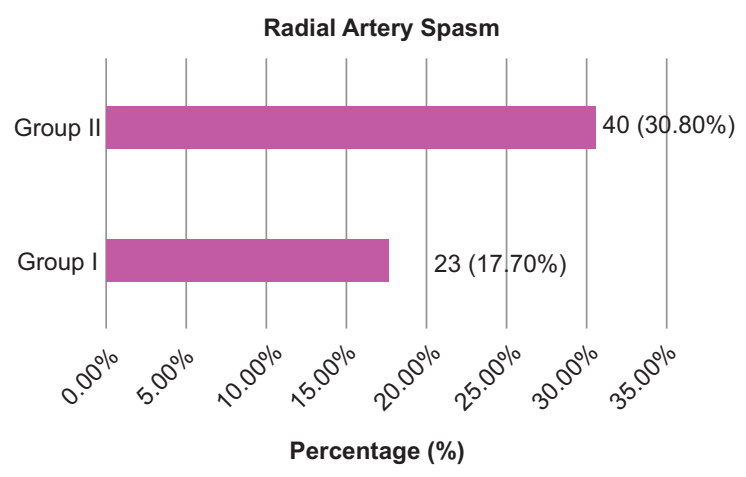

Fig.-2: Frequency of radial artery spasm

\section{Discussion:}

The principle finding of this prospective observational analytic study is the reduction in the frequency of radial artery spasm by administration of opioid analgesia during trans radial coronary intervention. This resulted in significant decrease in patient discomfort as well as access site crossover rate.
Mean age of the study population was of $55.88 \pm 7.22$ (SD) years with a majority in age group 51-60 years old. Clear male predominance was observed in both groups. No statistical difference was observed between two groups regarding age and gender distribution $(p>0.05)$. Shimu ${ }^{14}$ observed similar age and gender distribution. Mean BMI was $25.86 \pm 2.87 \mathrm{~kg} / \mathrm{m} 2$ in group I and $25.82 \pm 2.66 \mathrm{~kg} / \mathrm{m} 2$ in group II patients which correlate with the findings of the study. Mean height was $162.33 \pm 4.62 \mathrm{~cm}$ in group I and $162.82 \pm 4.50 \mathrm{~cm}$ in group II. $p$ valve was not significant between two groups considering BMI and height ( $\mathrm{p}>0.05$ ). In this study, $51.5 \%$ of group I and $50.80 \%$ of group II patients underwent PCI. No significant statistical difference noted between two groups $(p>0.05)$. In both groups, majority patients' access was right radial artery $(91.9 \%)$. Anatomical anomaly and subclavian tortuosity were found in $11.53 \%$ and $12.30 \%$ of group I patients and $10 \%$ and $7.5 \%$ of group II patients. Average anatomical anomaly was $10.76 \%$ which was similar to the study by Hassan ${ }^{15}$ where rate was $12.6 \%$. More than 2 catheters were used in $23.8 \%$ of group I patients and $33.8 \%$ of group II patients. No significant difference was observed between two groups regarding the use of number of catheters. New sheath was used in $45.76 \%$ and reprocessed sheath was used in $54.23 \%$ of patients with similar distribution in both groups. Length of procedure was $46.42 \pm 13.13 \mathrm{~min}$ in group I patients and 50.31 12.90 minutes in group II patients. The procedure was statistically significantly 
lengthy in group II patient ( $\mathrm{p}$ 0.017). VAS scale pain score was $4.75 \pm 2.70$ in group I and 5.33 \pm 2.64 in group II which was significantly less in group I patients (p 0.046). Selfreported patient discomfort was significantly lower in Group I. Spasm was, expectedly, strongly associated with higher patient discomfort. In group I, 12.30\% required repeat use of spasmolytic cocktail. In group II, $22.30 \%$ required spasmolytic cocktail. Group II patients needed more frequent usage of spasmolytic cocktail than group I (p 0.003). Multiple arterial prick was $53.8 \%$ in group I and $56.9 \%$ in group II with no significant difference ( $p$ 0.618). There was no significant statistical difference between the number of $5 \mathrm{Fr}$ and $6 \mathrm{Fr}$ catheter $(\mathrm{p}=.128)$ use.

The frequency of radial artery spasm was $30.80 \%$ in group II patients and $17.70 \%$ in group I patients. Negative association was found between radial artery spasm and use of fentanyl ( $\mathrm{p}$ 0.014). Patients' discomfort and pain was also significantly less among patients who received IV fentanyl. The number needed to treat (NNT) to avoid 1 case of spasm was 8 . Addition of fentanyl will reduce RAS $13 \%$ than standard treatment protocol. Deftereos ${ }^{16}$ found that NNT to avoid 1 spasm was 18 which was higher. They found spasm occurred in $2.6 \%$ patients of the treatment group versus $8.3 \%$ in the control group and RAS was labelled only by subjective measures. In this study, both subjective and or objective evidence were used to define RAS. So, the incidence of RAS was higher in both groups than the study done by Deftereos. ${ }^{16}$

Deftereos ${ }^{16}$ observed combined use of fentanyl and midazolam reduced radial artery spasm during coronary procedure through trans radial access. Minghui ${ }^{17}$ also observed significantly reduced radial artery spasm in coronary procedure through trans radial access. There is a hypothesis that spasm can be triggered by stress and pain, pre-medication with sedatives is being used but the effect of routine administration of sedation and analgesia on the incidence of radial artery spasm has not been systematically evaluated. ${ }^{8}$ Radial artery spasm complicates and prolongs the procedure, frustrates the interventionalist, increases the possibility of subsequent radial artery closure, aggravates patient discomfort \& pain and nullifies the advantages of the trans radial approach. In this study use of fentanyl showed significant reduction of radial artery spasm.

In bivariate logistic regression analysis, not using of fentanyl was observed having 4.3 times higher odds for occurring radial artery spasm in this study. PCI as procedure [ OR 2.614, 95\% CI (1.448-5.714), $\mathrm{p}<0.05$ ], female gender [OR 2.654, 95\% CI (1.470-4.791), p<0.05], diabetes mellitus [16.724, 95\% CI (7.866-35.560), $\mathrm{p}<0.05$ ], dyslipidemia [OR 4.680,95\% CI (2.454-8.927)], use of reprocessed sheath [OR 2.780, 95\% CI (1.2625.038), $\mathrm{p}<0.05$ ], VAS score during cannulation [OR $11.077,95 \%$ CI (4.031-21.1950), $\mathrm{p}<0.05]$, catheter number $>2$ [OR 9.133, 95\% (3.990-9.829), $\mathrm{p}<0.05$ ], catheter diameter (6Fr) [OR 2.073, 95\% CI (1.016-3.855), $\mathrm{p}<0.05]$, multiple arterial prick [OR 2.516, 95\% CI (1.403-2.532), $\mathrm{p}<0.05$ ], decreasing height of patient [ OR $.857,95 \% \mathrm{CI}(.773-.953), \mathrm{p}<0.05]$ and increasing duration of procedure [OR 6.657, 95\% CI (1.584-9.740), $\mathrm{p}<0.05$ ] had significantly higher odds for occurring radial artery spasm. Deftereos, et al., (2013) also did a univariate analysis and patients with shorter stature and lower body mass index were more prone to spasm along with female sex and smoking as also predisposing factors. They also found that spasm was more frequent in patients undergoing combined coronary angiography and PCI procedures and multiple number of catheter used/procedure. Increased duration of the procedure had higher odds for radial artery spasm. These findings corroborate with present study observation.

In multivariate logistic regression analysis, Not using of fentanyl [OR .370,95\% CI (.195-.703), p<0.05], catheter diameter 6Fr [OR 1.938, 95\% CI (1.118-1.99), p<0.05], use of reprocessed sheath [ OR 1.181, 95\% CI (1.349$4.121), \mathrm{p}<0.05$ ], smoking [ OR 2.230, 95\% CI 1.073$4.633, \mathrm{p}=.032]$ and VAS score during cannulation [OR $.988,95 \%$ CI $(.825-1.183), \mathrm{p}<0.05]$ were significant predictors of radial artery spasm. In the multivariate analysis, where the occurrence of spasm was entered as the dependent variable, the independent association of treatment with the incidence of spasm was tested after accounting for sex, height, diabetes mellitus, smoking, multiple arterial prick, number of catheter used/ procedure, procedure type (CAG and or PCI), use of reprocessed sheath and procedure duration. Deftereos ${ }^{16}$ also did multivariate analysis and found that patients with active smoking, short stature and low body mass index were more prone to spasm along with female gender. They also observed spasm was more frequent when number of catheter used was multiple and combined coronary angiogram and PCI was done. They denoted that the administration of sedation and analgesia was independently associated with a marked reduction in the incidence of spasm (multivariate hazard ratio: 0.140, 95\% CI: 0.073-0.271; $\mathrm{p}<0.001)$. These findings also corroborate with present study observation. 
Present study indicates that use of fentanyl reduces radial artery spasm among patients undergoing coronary intervention. Pre-medication with oral benzodiazepines has been introduced aiming to reduce spasm during arterial catheterization, but the effect of routine administration of analgesia on the incidence of radial artery spasm is still under-evaluated. So, the routine administration of analgesia in patients undergoing elective coronary intervention may led to a significant reduction in spasm occurrence.

\section{Limitations:}

All samples were collected from a single center (NICVD). Sample size was not representative to generalize the findings. Spasm cannot be always defined with objectivity. Definition used in study was quite restrictive because it required angiographic confirmation. Some patient subgroups were excluded. Findings might not apply to other sedative medications. The respondents who underwent CAG followed by PCI were generally considered as PCI procedure. Selection of the patients were operator's choice. Preprocedural duplex USG of radial artery was not done.

\section{Conclusion:}

In this study, use of fentanyl reduced the frequency of radial artery spasm and the procedure related level of patient discomfort.

\section{Recommendation:}

Radial artery spasm (RAS) is one of the obstacles of successful trans radial intervention. Some preventive measures could reduce the incidence of RAS including preoperative intravenous fentanyl. Strategies to prevent RAS and preserving radial artery should start from the patient selection with considering predictors of RAS. More similar studies are needed for strengthening the finding of our study. However, the result of this study needs further confirmation in a randomized large scale multi centre prospective cohort study.

\section{Conflict of Interest:}

None.

\section{References}

1. Mason PJ, Shah B, Tamis-Holland JE, Bittl JA, Cohen MG, Safirstein J, et al. An Update on Radial Artery Access and Best Practices for Transradial Coronary Angiography and Intervention in Acute Coronary Syndrome: A Scientific Statement from the American Heart Association. Circ Cardiovasc Interv. 2018;11: e000035.

2. Campeau L. Percutaneous radial artery approach for coronary angiography. Cathet Cardiovasc Diagn. 1989; 16:3-7.

3. Kiemeneij F, Laarman GJ, Melker E de. Transradial artery coronary angioplasty. Am Heart J. 1995; 129:1-7.
4. Bertrand OF, Larochellière R de, Rodés-Cabau J, Proulx G, Gleeton $\mathrm{O}$, Nguyen CM, et al. A randomized study comparing same-day home discharge and abciximab bolus only to overnight hospitalization and abciximab bolus and infusion after transradial coronary stent implantation. Circulation: American Heart Association. 2006;114: 2636-43.

5. Roussanov O, Wilson SJ, Henley K, Estacio G, Hill J, Dogan B, et al. Cost-effectiveness of the radial versus femoral artery approach to diagnostic cardiac catheterization. J Invasive Cardiol. 2007; 19: 349-53.

6. Caluk j. Procedural techniques of coronary angiography. In: Kirac S. Eds. Advances in the Diagnosis of Coronary Atherosclerosis: InTech. 2011: 95-120.

7. Caputo RP, Tremmel JA, Rao S, Gilchrist IC, Pyne C, Pancholy $\mathrm{S}$, et al. Transradial arterial access for coronary and peripheral procedures: executive summary by the Transradial Committee of the SCAI. Catheter Cardiovasc Interv. 2011;78: 823-39.

8. Ho HH, Jafary FH, Ong PJ. Radial artery spasm during transradial cardiac catheterization and percutaneous coronary intervention: incidence, predisposing factors, prevention, and management. Cardiovasc Revasc Med. 2012;13: 193-95.

9. Coppola J, Patel T, Kwan T, Sanghvi K, Srivastava S, Shah S, et al. Nitroglycerin, nitroprusside, or both, in preventing radial artery spasm during transradial artery catheterization. J Invasive Cardiol. 2006; 18: 155-58.

10. Chen C-W, Lin C-L, Lin T-K, Lin C-D. A simple and effective regimen for prevention of radial artery spasm during coronary catheterization. Karger Cardiology Journal. 2006; 105:43-47.

11. Ayzenberg S. Association of Radial Artery Spasm with Development of Radial Arterial Occlusion in Patients Undergoing Diagnostic Angiogram and/or Percutaneous Coronary Intervention (PCI) Via Radial Access: NCT02577796, 2014-09-19 [cited 2021 Mar 18]. Available from: https://clinicaltrials.gov/ct2/show/ NCT02577796.

12. Kristiæ I, Lukenda J. Radial artery spasm during transradial coronary procedures. J Invasive Cardiol. 2011;23: 527-31.

13. Jia D-A, Zhou Y-J, Shi D-M, Liu Y-Y, Wang J-L, Liu X-L, et al. Incidence and predictors of radial artery spasm during transradial coronary angiography and intervention. Chin Med J. 2010;123: $843-47$.

14. Shimu IJ, Alam MB, Islam AMM, Asaduzzaman K, Khan A, Khoda MME. Safety and Efficacy of Trans-Radial Percutaneous Coronary Intervention - Experience in a Tertiary Level Hospital of Bangladesh. Cardiovasc. j. 2020;13: 46-51.

15. Hassan AKM, Abdelmegid MA-KF, Hassan Ali H, Warda HM, Mahfouz RA, Tammam Alkhateeb AAA. Radial artery anomalies in patients undergoing transradial coronary procedures - An Egyptian multicenter experience. The Egyptian Heart Journal. 2016;68: 31-36.

16. Deftereos S, Giannopoulos G, Raisakis K, Hahalis G, Kaoukis A, Kossyvakis C, et al. Moderate procedural sedation and opioid analgesia during transradial coronary interventions to prevent spasm: a prospective randomized study. JACC Cardiovasc Interv. 2013;6: 267-73.

17. LI Minghui, BAI Yuan, TAN Hongwen, CHEN Xiang, XHANG Xhigang, QIN Yongwen, LI Minghui, BAI Yuan, TAN Hongwen, CHEN Xiang, XHANG Xhigang, et al. Effect of small dose of fentanyl combined with midazolam on prevention of radial artery spasm during transradial coronary intervention. sylcyyzz [Internet]. 2014:82-4. Available from: http://jcmp.yzu.edu.cn/en/article/doi/ 10.7619/jcmp.201409023. 